\section{Intestinal Ischaemia}

The incidence of acute intestinal infarction approximates to the reported death rate-some 600 deaths a year in the United Kingdom. Though it may be theoretically possible to restore the intestinal circulation, late diagnosis of cases with additional cardiac, respiratory, and renal abnormalities precludes a successful outcome in the majority, and the patient is usually doomed from the start.

Though the classical description is of a patient who develops sudden severe abdominal colic, passes blood and mucus per rectum, and rapidly develops peripheral circulatory failure, A. Marston ${ }^{1}$ rightly emphasizes that the mode of presentation is usually atypical. The onset is often insidious with only vague abdominal pain, and the patient may remain apparently well for hours or even days. At this stage, when the diagnosis must be made or laparotomy undertaken for treatment to be effective, the patient may have mild abdominal distension with generalized tenderness but no rigidity. Bowel sounds are normal or increased until the stage when peritonitis ensues, the abdomen is silent, and irreversible gangrene is present.

It requires a high degree of suspicion on the physician's part to consider a diagnosis of intestinal infarction at this stage, and the surgeon will often hesitate to undertake laparotomy for vague symptoms when the patient's general condition gives rise to doubts about the anaesthetic risk. As the ischaemic process continues, vast quantities of fluid and blood are lost, and the patient rapidly deteriorates. Marston points out that, when florid physical signs have appeared, the point of recovery for the patient has probably been passed.

Investigation of the acute case yields disappointing results. Plain films of the abdomen may show intestinal distension, and aortography is of doubtful value. Leucocytosis occurs early and may be of value, but other laboratory investigations merely reflect the severity of the underlying changes.

Before surgical operation resuscitation by intravenous fluids, correction of metabolic acidosis by bicarbonate, and administration of antibiotic may help to prepare the patient. Administration of heparin is unlikely to be rewarding. Though total midgut necrosis is lethal, it is sometimes possible to undertake a limited resection, the removal of a mesenteric embolus by Fogarty catheter, or revascularization of the superior mesenteric artery by bypass graft or by direct anastomosis to aorta or common iliac. Routine reoperation 24 hours later is strongly advised to assess the success of revascularization and to resect any bowel which has subsequently become gangrenous.

Chronic intestinal ischaemia is a different problem, but, if the diagnosis can be made, successful revascularization may avert acute infarction. The classical history of severe, persistent, postprandial pain ("intestinal angina"), vomiting, severe loss of weight, and alteration in bowel habit is seen only infrequently, and the patient is more likely to present with vague abdominal pain and moderate loss of weight. Early enthusiasm for the investigation and treatment of cases due to atherosclerosis has waned because many patients develop a good collateral blood supply and do not require surgery. ${ }^{2}$

Recently attention has been paid to the coeliac axis compression syndrome. American reports ${ }^{3}$ are encouraging in that patients with abdominal pain, weight loss, and bowel upset have been shown to have stenosis of the coeliac axis caused by external compression from the median arcuate ligament of the diaphragm or from periarterial fibrosis. Simple division of the constricting fibres brings relief of symptoms in $70-80 \%$ of cases. J. A. Edwards and his colleagues ${ }^{4}$ are not so convinced, and there is certainly a need for further study of this problem.

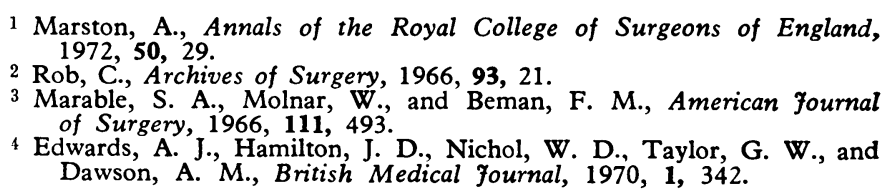

\section{Smoking and Vascular Disease}

Despite the recent report of the Royal College of Physicians $^{1}$ there has been little change in British smoking habits. The medical profession is the only professional group in which cigarette smoking has lessened, and the decline in mortality from lung cancer among doctors is highly significant. ${ }^{2}$

Now the harmful association between smoking and peripheral vascular disease is becoming increasingly well documented. In $1960 \mathrm{~J}$. L. Juergens and his colleagues ${ }^{3}$ at the Mayo Clinic reviewed 520 patients known to have had arterial occlusion for five years or more, and showed that none who gave up smoking subsequently required amputation. Of those who continued smoking $11.4 \%$ required amputation for gangrene. The evidence incriminates cigarette smoking as a major cause of deterioration in patients with established arterial occlusive disease. R. Wray and his colleagues $^{4}$ found that in 45 aortofemoral bypass grafts nine patients who continued to smoke developed late occlusion of their grafts and none of the 16 who stopped smoking developed occlusion within a three to six years follow-up period. There is therefore good reason to advise patients with arterial occlusive disease to stop smoking.

Though these findings do not necessarily incriminate smoking as an aetiological factor in the development of disease the inference is strong. Apart from the accepted causal relationship between smoking and Buerger's disease, there is evidence of a strong association between arterial disease due to atherosclerosis and cigarette smoking. Few patients with intermittent claudication or gangrene are nonsmokers, and many of the latter are elderly or suffer from diabetes mellitus or embolism. Few would assert, as $\mathrm{H}$. $\mathrm{H}$. G. Eastcott ${ }^{5}$ does, that he saw fewer than 20 male nonsmokers in over 2,000 cases, but analysis of male patients attending any vascular clinic will show consistently that well over $95 \%$ are smokers-a proportion which is inconsistent with the distribution of smokers in the general population.

In a careful and well documented necropsy study of aortic atheroma in 1,019 patients who died from cancer and who had been asked about their smoking habits during life, D. L. Sackett and his colleagues ${ }^{6}$ showed a statistically significant increase in the severity of atheroma in smokers compared with non-smokers. Ex-smokers showed a pattern similar to that of non-smokers, and there was no significant difference between those who regularly drank alcohol and 